\title{
CONVEXITY PRESERVING SUMMABILITY MATRICES
}

\author{
C.R. SELVARAJ \\ Pennsylvania State University \\ Shenango Valley Campus \\ 147 Shenango Avenue \\ Sharon, PA 16146 \\ (Received February 28, 1989 and in revised form May 27, 1989)
}

ABSTRACT. The main result of this paper gives the necessary and sufficient conditions for the Abel matrices to preserve the convexity of sequences. Also, the higher orders of the Cesaro method are shown to be convexity-preserving matrices.

KEY WORDS AND PHRASES. Convex sequences, convexity-preserving matrices, domain of a matrix.

1980 AMS SUBJECT CLASSIFICATION CODE. 40

1. INTRODUCTION.

A real sequence $\left(x_{n}\right)_{n=0}^{\infty}$ is called convex if its second order differences

$$
\Delta^{2} x_{n}=x_{n+2}-2 x_{n+1}+x_{n}>0
$$

for $n=0,1,2, \ldots$.

G.H. Toader [1] proved the following two theorems characterizing the convex sequences.

THEOREM 1.1. If the sequence $\left(x_{k}\right)$ is given by

$$
x_{k}=\sum_{i=0}^{k}(k-i+1) b_{i},
$$

then $\Delta^{2} x_{k}=b_{k+2}$.

THEOREM 1.2. The sequence $\left(x_{k}\right)$ is convex if and only if $b_{k}>0$ for $k>2$ where $b_{k}$ 's are given by $(1.2)$.

The aim of the next section is to prove the necessary and sufficient conditions for the Abel matrix to preserve the convexity of sequences, using the above theorems. 
2. CONVEXITY PRESERVATION OF ABEL MATRIX.

For each sequence $\left(t_{n}\right)$ satisfying $0<t_{n}<1$ and 1 im $t_{n}=0$, the Abel matrix $A_{t}$ (Fridy [2, p. 421]) is defined by

$$
A_{t}[n, k]=t_{n}\left(1-t_{n}\right)^{k}
$$

In [2], Fridy gives the characterization of the domain of the matrix $A_{t}$. $A$ sequence $x$ is in the domain of $A_{t}$ if and only $\underset{k}{\lim } \underset{\mathrm{i}}{\operatorname{li}}\left|\mathrm{x}_{k}\right|^{1 / k}<1$. It follows that all convex sequences need not be in the domain of $A_{t}$. An obvious example is the convex sequence $x$ given by $x_{k}=k^{k}$ for $k=1,2,3, \ldots$. So, we restrict ourselves to the convex sequences that are in the domain of the matrix.

THEOREM 2.1. The Abel matrix $A_{t}$ preserves the convexity of the sequences that are in the domain of $A_{t}$ if and only if for all $n=0,1,2, \ldots$,

$$
\begin{aligned}
& \text { (a) } \Delta^{2} y_{n}(1)=0 \text { and } \\
& \text { (b) } \Delta^{2} y_{n}(k)>0 \text {, for } k=2,3, \ldots \text {, }
\end{aligned}
$$

where for each $k=0,1,2,3, \ldots$, the sequence $\left\{y_{n}(k)\right\}_{n=0}^{\infty}$ is defined by

$$
y_{n}(k)=\frac{\left(1-t_{n}\right)^{k}}{t_{n}} \text {. }
$$

PROOF. It is useful to note that

$$
\Delta^{2} y_{n}(1)=\Delta^{2} y_{n}(0)
$$

First we prove a general result for a convex sequence in terms of the sequences $\left\{y_{n}(k)\right\}_{n=0}^{\infty}$. If $x$ is a convex sequence in the domain of $A_{t}$, then the $n t h$ term of the transformed sequence is given by

$$
\begin{aligned}
\left(A_{t} x\right)_{n} & =t_{n} \sum_{k=0}^{\infty}\left(1-t_{n}\right)^{k} \sum_{1=0}^{k}(k-1+1) b_{1} \text {, using (1.2) } \\
& =t_{n} \sum_{i=0}^{\infty} b_{1} \sum_{k=1}^{\infty}\left(1-t_{n}\right)^{k}(k-1+1) .
\end{aligned}
$$

The change in the order of summation is justified as follows:

$$
\begin{aligned}
& \sum_{k=0}^{\infty} \sum_{i=0}^{k}\left|b_{i}\left(1-t_{n}\right)^{k}(k-1+1)\right| \\
& =\left(A_{t} x\right)_{n}+\left(\left|b_{0}\right|-b_{0}\right) \sum_{k=0}^{\infty}(k+1)\left(1-t_{n}\right)^{k}+\left(\left|b_{1}\right|-b_{1}\right) \sum_{k=0}^{\infty} k\left(1-t_{n}\right)^{k},
\end{aligned}
$$

and since $x$ is in the domain of $A_{t}$ and $1-t_{n} \varepsilon(0,1)$ for all $n$, the right-hand side of the equation (2.3) converges. Therefore from (2.2),

$$
\left(A_{t} x\right)_{n}=\sum_{1=0}^{\infty} b_{1} \frac{\left(1-t_{n}\right)^{1}}{t_{n}} \text {. }
$$


It follows that for $n=0,1,2, \ldots$,

$$
\Delta^{2}\left(A_{t} x\right)_{n}=\sum_{i=0}^{\infty} b_{i}\left[\frac{\left(1-t_{n+2}\right)^{i}}{t_{n+2}}-\frac{2\left(1-t_{n+1}\right)^{i}}{t_{n+1}}+\frac{\left(1-t_{n}\right)^{i}}{t_{n}}\right] \text {. }
$$

Using the equation (2.1), we can write the above equation as follows:

$$
\Delta^{2}\left(A_{t} x\right)_{n}=\left(b_{0}+b_{1}\right) \Delta^{2} y_{n}(1)+\sum_{k=2}^{\infty} b_{k} \Delta^{2} y_{n}(k)
$$

Now, assume that conditions (a) and (b) given in the theorem are true. Then the equation (2.4) together with Theorem 1.2 implies that

$$
\Delta^{2}\left(A_{t} x\right)_{n}>0
$$

Hence, the sequence $A_{t} x$ is convex.

Conversely, assume that the matrix $A_{t}$ preserves convexity of the sequences in its domain. Suppose that the condition (a) fails to hold. Then there exists a nonnegative integer $N$ such that $\Delta^{2} y_{N}(1)=L \neq 0$. If $L>0$, consider the convex sequence $u=\{-1,-2,-3, \ldots\}$, which is in the domain of $A_{t}$. It is easy to verify that any sequence $\left(x_{k}\right)$ may be written as in equation (1.2) by taking

$$
b_{0}=x_{0} \quad \text { and } \quad b_{k}=x_{k}-\sum_{1=0}^{k-1}(k-1+1) b_{1}, \quad \text { for } k>1 \text {. }
$$

Thus for the above sequence $u$,

$$
b_{0}=-1 \text { and } b_{k}=0 \text {, for } k>1 \text {. }
$$

Substituting these values of b's into the equation (2.4) we get

$$
\Delta^{2}\left(A_{t} u\right)_{N}=-\Delta^{2} y_{N}(1)=-L<0,
$$

which contradicts that the transformed sequence $A_{t} u$ is convex.

If $\mathrm{L}<0$, we repeat a similar argument considering the convex sequence $v=\{1,2,3, \ldots\}$ to get a contradiction. Hence, if the matrix $A_{t}$ preserves the convexity of sequences, then the condition (a) is true.

Next, suppose that the condition (a) is true, but not (b). Then there exists a non-negative integer $j$ such that the sequence $\left\{y_{n}(j)\right\}_{n=0}^{\infty}$ is not convex. That is, for some $N$,

$$
\Delta^{2} y_{N}(j)<0
$$

Now, construct a convex sequence $x$ as follows:

$$
x_{j}=\frac{-\left(1-t_{N+2}\right) j}{t_{N+2}}, x_{j-1}=\frac{-\left(1-t_{N+1}\right) j}{t_{N+1}}, x_{j-2}=\frac{-\left(1-t_{N}\right)^{j}}{t_{N}}
$$

and choose the other terms of the sequence $\left(x_{k}\right)$ in such a way that $\Delta^{2} x_{k}=b_{k+2}=0$ for al1 $k \neq j-2$. By construction,

$$
\Delta^{2} x_{j-2}=b_{j}>0 \text {, by }(2.6) \text {. }
$$


Using these values of $b_{k}$ 's together with our assumption (a) in equation $(2.4)$ for $n=$ $\mathrm{N}$, we get

$$
\Delta^{2}\left(A_{t} x\right)_{N}=b_{j} \Delta^{2} y_{N}(j)<0,
$$

which again contradicts that $A_{t} x$ is a convex sequence. This completes the proof.

We give below an example of an Abel matrix $A_{t}$ which preserves the convexity of sequences.

EXAMPLE 2.1. The Abel matrix $A_{t}$ where the sequence $\left(t_{n}\right)$ is given by

$$
t_{n}=\frac{1}{n+2} \text {, for } n=0,1,2, \ldots
$$

preserves the convexity of sequences. We shall show that this matrix $A_{t}$ satisfies the conditions (a) and (b) asserted in Theorem 2.1. Since

$$
\begin{aligned}
& y_{n}(1)=\frac{1-t_{n}}{t_{n}}=n+1, \\
& \Delta^{2} y_{n}(1)=0 .
\end{aligned}
$$

In order to see that the condition (b) is satisfied, for each $k=2,3, \ldots$, we have

$$
\Delta^{2} y_{n}(k)=\frac{(n+3)^{k}}{(n+4)^{k-1}}-\frac{2(n+2)^{k}}{(n+3)^{k-1}}+\frac{(n+1)^{k}}{(n+2)^{k-1}} .
$$

It suffices to prove that $f(n+1)>f(n)$ where

$$
f(n)=\frac{(n+2)^{k}}{(n+3)^{k-1}}-\frac{(n+1)^{k}}{(n+2)^{k-1}} .
$$

By differentiation it can be easily proved that $f(n)$ is an increasing function.

\section{PRESERVING CONVEXITY BY CESARO.}

In this section we use Theorems 1.1 and 1.2 to prove that the higher orders of Cesaro means preserve the convexity of sequences. In order to prove this result, we introduce some notations and terminologies. Let $K$ represent the set of all real convex sequences.

DEFINITION 3.1. For a summability matrix $A$ we define the set $K(A)$ as the set of all sequences such that the transformed sequences are convex, 1.e.,

$$
K(A)=\{x: A x \in K\}
$$

DEFINITION 3.2. The matrix $A$ is $K$-included by the matrix B provided that $K(A) \subseteq K(B)$.

DEFINITION 3.3. The matrix $B$ is $K$-stronger than the matrix $A$ provided that $K(A) \underset{\not}{\subsetneq} K(B)$.

Before we prove the main result we state the following preliminary theorems which are easy to prove. 
THEOREM 3.1. Let $A$ be a row-finite matrix and $T$ be a triangle (lower triangular matrix with non-zero dlagonal entries). Then the matrix $\mathrm{AT}^{-1}$ preserves the convexity of sequences if and only if $T$ is $K-1$ ncluded by $A$.

THEOREM 3.2. Let $A$ be a row-finite matrix and $T$ be a triangle. Then the matrix $\mathrm{AT}^{-1}$ is $\mathrm{K}$-stronger than the identity matrix if and only if $\mathrm{A}$ is $\mathrm{K}$-stronger than $\mathrm{T}$.

For $j \varepsilon \mathbb{N}$, the $j$ th order of the Cesáro matrix is given by Hardy [3, pp. 96-98] as

$$
C_{j}[n, k]= \begin{cases}\frac{\left(\begin{array}{c}
n-k+j+1 \\
j-1
\end{array}\right)}{\left(\begin{array}{c}
n+j \\
j
\end{array}\right)}, & \text { if } k<n, \\
0, & \text { if } k>n .\end{cases}
$$

In [1], G.H. Toader proved that the Cesaro matrix $C_{1}$ is K-stronger than the identity matrix.

THEOREM 3.3. For each $j \in \mathbb{I N}$, the matrix $\mathrm{C}_{j+1} \mathrm{C}_{j}^{-1}$ preserves the convexity of sequences.

PROOF. If $C_{j+1} C_{j}^{-1}$ is represented by $A_{j}$, it is easy to see that

$$
A_{j}[n, k]= \begin{cases}\frac{\left(\begin{array}{c}
j+k \\
j
\end{array}\right)}{\left(\begin{array}{c}
n+j+1 \\
j+1
\end{array}\right)} & \text { if } k<n, \\
0, & \text { if } k>n .\end{cases}
$$

Let $\mathbf{x}$ be a convex sequence. Then

$$
\left(A_{j} x\right)_{n}=\sum_{k=0}^{n} A_{j}[n, k] x_{k}
$$

A simple calculation shows that

$$
\left(\begin{array}{c}
j+n \\
j
\end{array}\right) x_{n}=\left(\begin{array}{c}
n+j+1 \\
j+1
\end{array}\right)\left(A_{j} x\right)_{n}-\left(\begin{array}{c}
n+j \\
j+1
\end{array}\right)\left(A_{j} x\right)_{n-1} .
$$

Writing

$$
\left(A_{j} x\right)_{n}=\sum_{i=0}^{n}(n-1+1) e_{1}
$$

we can see that the equation (3.1) can be simplified to

$$
x_{n}=\frac{1}{j+1} \sum_{i=0}^{n}[n+(j+1)(n-i+1)] e_{i} \cdot
$$

Therefore for each $n=0,1,2, \ldots$,

$$
\Delta^{2} x_{n}=\frac{1}{j+1}\left[(n-3+j) e_{n+2}-n e_{n+1}\right],
$$

which implies that

$$
(n-3+j) e_{n+2}=n e_{n+1}+(j+1) \Delta^{2} x_{n} \text {. }
$$


Since $x$ is a convex sequence, it follows from Theorem 1.2 that

$$
\Delta^{2} x_{n}=b_{n+2}>0 \text { for } n=0,1, \ldots \text {. }
$$

Using this fact in the equation (3.3), we obtain

$$
e_{n+2}>0 \text { for } n=0,1, \ldots \text {. }
$$

Hence, the equation (3.2) together with (3.4) implies that [cf. Theorem 1.2] the transformed sequence $A_{j} x$ is convex. This completes the proof.

REMARK 3.1. Combining the theorems 3.1 and 3.3 we see that

$$
k\left(c_{1}\right) \subseteq k\left(c_{2}\right) \subseteq \cdots \subseteq k\left(c_{j}\right) \subseteq k\left(c_{j+1}\right) \subseteq \cdots
$$

Since the Cesáro matrix $C_{1}$ preserves the convexity of sequences [1], 1t follows that for each $j \varepsilon \mathbb{I N}$ the $j$ th order of the Cesaro matrix $C_{j}$ preserves the convexity. Also, by considering the sequence $x=\{0,1,0,0, \ldots$,$\} which is not convex, it is easy to prove$ that

$$
\Delta^{2}\left(A_{j} x\right)_{n}>0
$$

Thus the matrix $\mathrm{C}_{j+1} \mathrm{C}_{j}^{-1}$ is $\mathrm{K}$-stronger than the identity matrix.

Therefore

$$
\mathrm{k}\left(\mathrm{C}_{1}\right) \varsubsetneqq \mathrm{k}\left(\mathrm{C}_{2}\right) \varsubsetneqq \ldots \varsubsetneqq \mathrm{k}\left(\mathrm{C}_{j}\right) \varsubsetneqq \mathrm{K}\left(\mathrm{C}_{j+1}\right) \varsubsetneqq \ldots
$$

Now we state this result in the following theorem.

THEOREM 3.4. For $j \in \mathbb{N}$, the $j$ th order Cesaro matrix $C_{j}$ preserves the convexity of the sequences. Also, $C_{j+1}$ is $\mathrm{K}$-stronger than $C_{j}$, which is $\mathrm{K}$-stronger than the identity matrix.

REMARK 3.2. The Hơlder's summability matrix of order $j$ is given by

$$
H_{j}=\left(c_{1}\right)^{j} \text { for } j=1,2, \ldots \text {. }
$$

Therefore the matrix $\mathrm{H}_{\mathrm{j}}$ preserves the convexity of the sequences and also the matrix $\mathrm{H}_{j+1}$ is $\mathrm{K}-\mathrm{s}$ tronger than the matrix $\mathrm{H}_{j}$.

\section{REFERENCES}

1. TOADER, G.H., A Hierarchy of Convexity for Sequences, L'anal. Num. et la Th. de 1 'approx. 12 (1983), 187-192.

2. FRIDY, J.A., Abel Transformations into $\ell^{1}$, Canad. Math. Bull. 25 (1982), 421-427.

3. HARDY, G.H., Divergent Series, Oxford University Press, London, 1949. 


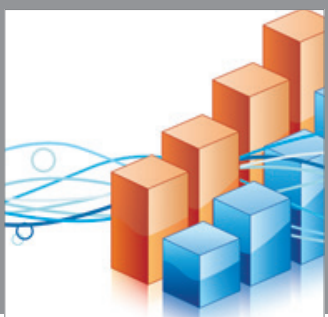

Advances in

Operations Research

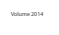

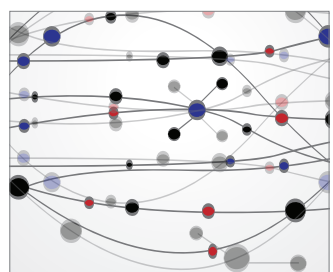

\section{The Scientific} World Journal
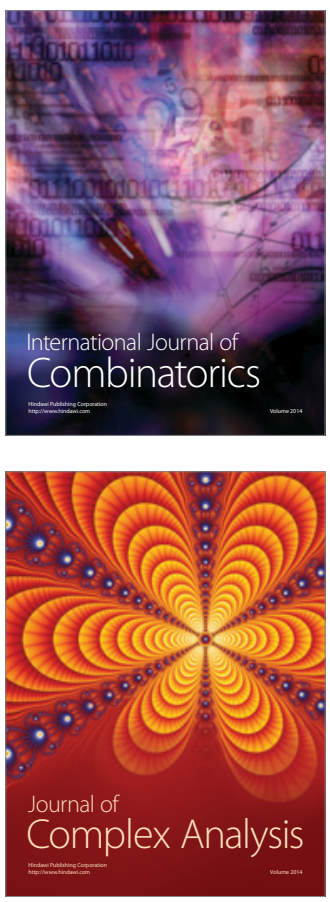

International Journal of

Mathematics and

Mathematical

Sciences
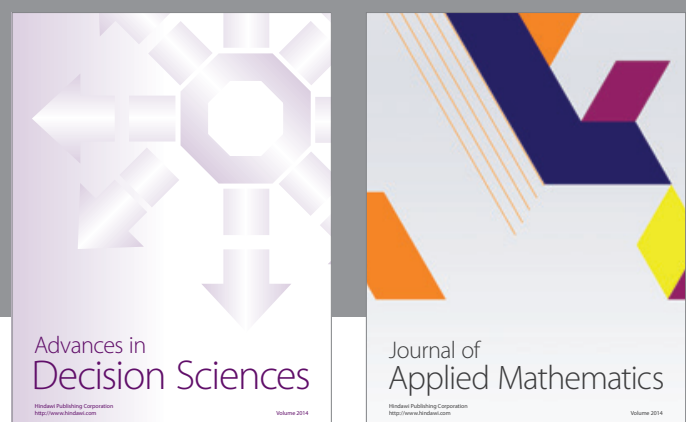

Journal of

Applied Mathematics
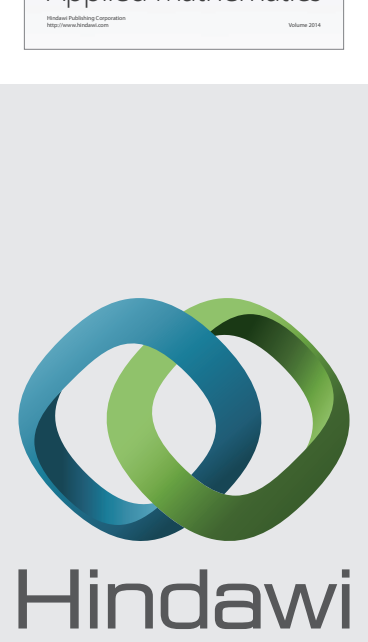

Submit your manuscripts at http://www.hindawi.com
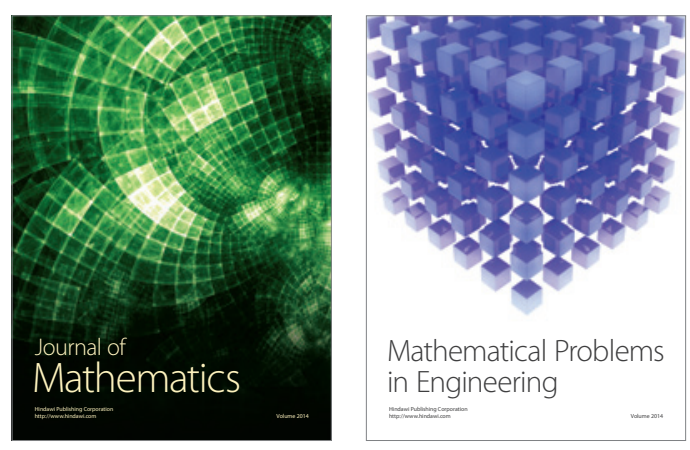

Mathematical Problems in Engineering
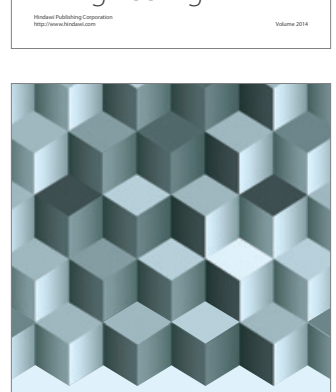

Journal of

Function Spaces
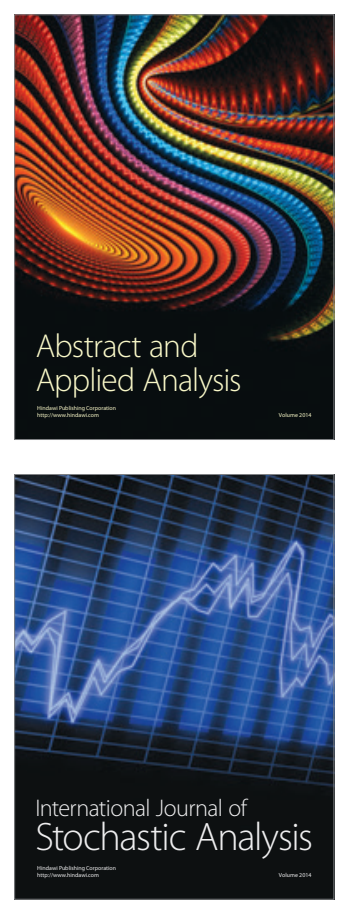

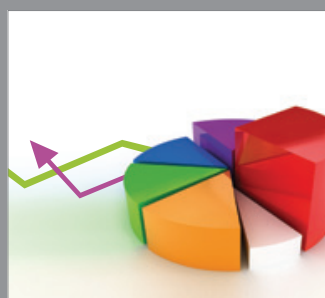

ournal of

Probability and Statistics

Promensencen
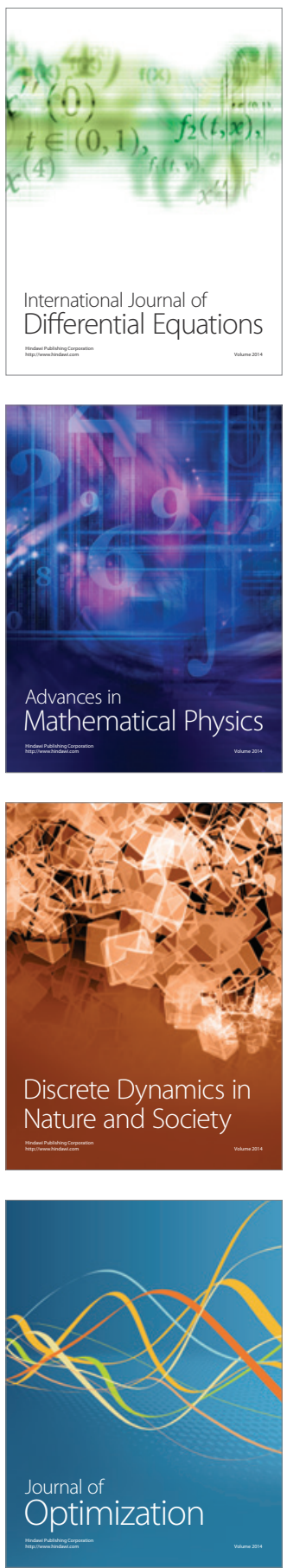